\title{
ILCEA
}

Revue de l'Institut des langues et cultures

d'Europe, Amérique, Afrique, Asie et Australie

$20 \mid 2014$

Le conte : d'un art à l'autre

\section{Les Enfants de Poucet: Avatars du conte dans le théâtre francophone contemporain (1989-2012)}

Little Thumbling's Children: The Avatars of Tale in Francophone Theatre (19892012)

\section{Martial Poirson}

\section{OpenEdition}

\section{Journals}

Édition électronique

URL : http://journals.openedition.org/ilcea/2798

DOI : 10.4000/ilcea.2798

ISSN : 2101-0609

Éditeur

UGA Éditions/Université Grenoble Alpes

Édition imprimée

ISBN : 978-2-84310-290-5

ISSN : 1639-6073

Référence électronique

Martial Poirson, « Les Enfants de Poucet : Avatars du conte dans le théâtre francophone

contemporain (1989-2012) », ILCEA [En ligne], 20 | 2014, mis en ligne le 08 décembre 2014, consulté le 01 mai 2019. URL : http://journals.openedition.org/ilcea/2798 ; DOI : 10.4000/ilcea.2798

Ce document a été généré automatiquement le 1 mai 2019.

(c) ILCEA 


\title{
Les Enfants de Poucet : Avatars du conte dans le théâtre francophone contemporain (1989-2012)
}

Little Thumbling's Children: The Avatars of Tale in Francophone Theatre (19892012)

\author{
Martial Poirson
}
« Poucet est un héros parce qu'il sait traverser le sevrage et vivre alors l'incomplétude de la vie, incomplétude qui permet le déploiement d'un espace mental et imaginaire. » (Joël Pommerat à propos de la mise en scène du Petit Poucet de Caroline Baratoux par Pascale Nandillon ${ }^{1}$.)

1 Ce n'est pas le moindre des paradoxes du théatre contemporain que de puiser dans le répertoire du conte une part non négligeable de son inspiration: revendiquant une rupture par rapport à la tradition, il se nourrit pourtant de ce qui peut à bon droit apparaître comme la quintessence du patrimoine culturel européen, à la fois littéraire et immatériel, issu de la transmission d'artistes-conteurs. Au sein d'une telle configuration esthétique et idéologique, le conte-source du « Petit Poucet » est sans doute l'un des plus fréquemment adaptés en ce début de $\mathrm{xxI}^{\mathrm{e}}$ siècle, depuis le théâtre jeunesse jusqu'aux nouvelles écritures scéniques, au point qu'il est permis d'évoquer un « effet Petit Poucet » propre aux "adaptations-recréations» de Perrault (Bernanoce, 2008: 140). Renouant avec la ferveur qu'il a connu au cours du XvIII ${ }^{\mathrm{e}}$ siècle (Poirson, $\left.2011: 133-175 ; 2009\right)$ en mettant fin à son éclipse relative au cours des $\mathrm{XIX}^{\mathrm{e}}$ et surtout $\mathrm{XX}^{\mathrm{e}}$ siècles (Martin, 2007 ; Laplace-Claverie, 2007), au profit de «Cendrillon », de « Riquet à la Houppe » ou du « Petit Chaperon rouge », la transposition théâtrale du «Petit Poucet " trouve depuis une vingtaine d'années un second souffle dans l'écriture dramatique contemporaine francophone (Belgique, Suisse, Québec) aussi bien que dans les nouvelles expérimentations spectaculaires. Pourtant, contrairement aux adaptations 
cinématographiques qui leur sont contemporaines ${ }^{2}$, rares sont les réécritures dramatiques récentes réellement explicites et suivies du "Petit Poucet », bien qu'il soit, de façon incidente, extrêmement présent à la scène. Le plus souvent, la profusion des adaptations contraste fortement avec le minimalisme dramaturgique de la transposition, qui parfois se réduit à un simple effet de citation, même s'il revêt souvent une valeur matricielle au sein du système dramaturgique ou scénographique. Parfois même, on assiste à un double mouvement apparemment contradictoire de prise de distance et d'exemplification du conte-source.

2 Cependant, si la tradition a eu tendance à voir dans le conte-source un récit d'ascension et à proposer des transpositions soucieuses avant tout de rendre exemplaire le geste héroïque de l'enfant infirme ou mal doté mais néanmoins valeureux, c'est au contraire au prix d'un renversement de perspective que Poucet, de progéniture, devient géniteur : le passage au théâtre permet un basculement du récit initiatique de formation vers la fable de transmission. Il féconde toutes sortes d'avatars en rupture avec leurs origines. C'est ainsi qu'un certain nombre de propositions artistiques se plaisent à mélanger les âges et les genres, à l'instar de Laurent Gutmann avec le Petit Poucet (2012) ${ }^{3}$, selon lequel l'acteur « incarnant le Petit Poucet sera nettement plus âgé » que les couples « jeunes et pleins de santé » (la distribution associe le couple démissionnaire des bûcherons au couple prédateur des ogres), «comme un éternel enfant dont on ne sait que faire, un presque vieillard inutile dont on se débarrasse parce qu'on en a un peu honte ». C'est aussi la stratégie de José Pliya dans Mon Petit Poucet (2012), sans doute influencé par la mythologie africaine de l'«Enfant roi $^{4}$ ", qui afin de procéder à la "déconstruction de l'autorité paternelle ${ }^{5}$ " renverse le rapport asymétrique parent-enfant en montrant un père dépassé, cherchant en vain à retrouver un fils « rentré tout seul comme un grand ».

Un tel changement de paradigme est lourd de conséquences, aussi bien en termes de structure dramaturgique que de choix esthétiques et idéologiques. D'abord, la figure de Poucet, longtemps hyper-individualisée, apte à prendre en charge l'organisation de l'intrigue, devient beaucoup plus diffuse au sein de distributions flottantes, cependant que se multiplient les effets de focalisation, tantôt sur le point de vue du père ou de la mère, tantôt sur celui de l'ogre ou de sa femme, voire de ses filles. Ensuite, la portée axiologique ou éthique du conte-source est de façon assez systématique évacuée ou ironisée, voire franchement déconstruite, comme le suggérait Perrault lui-même, au profit de considérations tantôt psychologiques (développement psychique de l'adolescent, sevrage affectif, entrée dans l'âge adulte), tantôt psychanalytiques (complexe d'infériorité, syndrome d'abandon, pulsion de mort), tantôt sociologiques (se soustraire à la reproduction sociale, échapper au déterminisme culturel, s'élever audessus de sa condition de bûcheron, conjurer l'hérédité sociale), tantôt existentielles ou métaphysiques (devenir acteur de sa propre vie, échapper à l'incomplétude, poursuivre la quête du sens). C'est la source de stratégies d'actualisation et de déterritorialisation du conte, curieusement transposé, tantôt dans le monde le plus contemporain, tantôt dans les aires culturelles les plus diverses. Encore est-ce parfois au prix d'une certaine dévitalisation de la visée proprement politique du conte, au profit de l'investissement de questions sociétales, familiales et domestiques actuelles liées à l'enfance: boulimie, abandon, solitude, confrontation à la violence, quête du père ou de la mère... Autrement dit, la transposition théâtrale procède à un triple mouvement d'atténuation du substrat mythogène du conte, d'affirmation d'une mythologie personnelle de l'enfant abandonné et d'exploitation de situations dramatiques inspirées par les structures névrotiques du 
roman familial. Cependant, même dans ces formes dégradées et appauvries du matériau symbolique ou anthropologique, la finalité idéologique du conte est loin d'être perdue de vue. Envisagée par la tradition herméneutique comme une parabole de l'émancipation des subalternes, la fable est donc aujourd'hui essentiellement perçue comme une métaphore de l'aliénation, le plus souvent volontaire, voire des conditionnements de tous ordres dont le personnage éponyme est susceptible - ou non - de chercher à s'affranchir - parfois en vain. L'ampleur du motif de la dévoration dans la plupart des réécritures récentes est sans doute le signe le plus évident de cette transformation.

4 En dernier lieu, l'univers de référence du conte-source est de plus en plus fréquemment mis à distance au profit de l'amplification de motifs isolés, de détails significatifs ou de séquences emblématiques directement identifiables par le public. Jouant sur la connivence culturelle avec le spectateur, le théâtre se démarque des traditionnels effets merveilleux appuyés pour rendre la fable prosaïque, voire triviale, ou à l'inverse pour en proposer une affabulation poétique, dramaturgique ou scénographique. Longtemps fondée sur l'adaptation la plus fidèle et scrupuleuse du conte-source, dont on reprenait la trame narrative, le système des personnages, les ressorts merveilleux et jusqu'aux effets de théâtre inhérents au conte lui-même, la transposition se caractérise au contraire dans le théâtre contemporain par une dilution de la fable, souvent réduite à de simples effets de citation verbale ou visuelle; parfois même, elle paraît totalement implicite dans la pièce, comme dans En attendant le Petit Poucet (2001) de Philippe Dorin, en dépit d'un titre idiosyncratique qui se joue de l'horizon d'attente du lecteur ou du spectateur. En opérant une profération orale du conte littéraire qui est aussi un retour aux sources du conte traditionnel, le théâtre concourt ainsi à une certaine autonomisation de la posture conteuse, qui puise largement dans les façons de faire et de dire des artistes-conteurs et dans les techniques des arts du récit, dont les frontières avec les arts du spectacle sont particulièrement poreuses (Poirson, 2012).

5 La structure de l'écriture dramatique se ressent d'une telle évolution: les transpositions théâtrales tendent vers une forme d'épicisation où le récit fait retour sur le plateau, soit par sa prise en charge par un récitant extérieur à la diégèse, soit par l'intermédiaire d'une voix off, soit par le truchement d'un personnage exposant, sous forme d'adresse directe au spectateur ou d'hypotypose, ce qui généralement se joue hors scène. Une voix didascalique se substitue alors en partie au dialogue, voire à l'action, avec laquelle elle introduit un écart, tout en faisant de la pratique de réécriture la finalité même de la dramaturgie. Un certain nombre de pièces font en outre émerger une structure chorale où tantôt les qualités paradoxales de bravoure de Poucet, tantôt l'instinct démesuré de dévoration de l'ogre peuvent être partagés, voire assumés par plusieurs personnages différents. Plus que jamais autoréférentiel, le conte dramatique exacerbe par conséquent la théâtralité du conte-source et rend sensible sa propre dimension métathéâtrale, guidant la réflexion sur ses procédés de production de l'illusion scénique et de réception du pacte de créance ${ }^{6}$.

6 En interrogeant une douzaine de transpositions du conte-source mises en spectacle et parfois, mais pas systématiquement, mises en texte depuis une vingtaine d'années, on cherchera à expliciter un certain nombre d'impératifs dramaturgiques et scénographiques qui s'offrent aujourd'hui aux auteurs et metteurs en scène. Il s'agira d'envisager la façon dont la transposition théâtrale, en s'affranchissant des règles de l'adaptation dramatique du conte-source, en propose une forme d'actualisation, voire de déterritorialisation dysfonctionnelle susceptible d'en exacerber la teneur politique et 
idéologique, mais également d'en réamorcer la charge symbolique, tout en révélant les potentialités dramaturgiques et scénographiques du conte.

\section{S'affranchir des règles de l'adaptation dramatique du conte-source}

Il est frappant de constater à la fois l'omniprésence de la figure de Poucet dans le théâtre francophone (Bernanoce, 2009: 209-229), et conjointement son imprégnation diffuse, source d'adaptations sciemment infidèles ou de transpositions ostensiblement impures, voire inavouées. Il existe en effet, dans le répertoire contemporain, relativement peu d'exemples d'adaptations proprement dites du conte, à l'instar de celle de Caroline Baratoux dans Le Petit Poucet (2008), qui en suit assez fidèlement l'intrigue : à partir de la trame narrative de Perrault, elle fait saillir le vertige de l'expérience limite de la faim et du sevrage qui contraste avec l'appétit gigantesque de l'ogre, la confrontation à la violence du père et à l'impuissance de la mère, le rite initiatique de passage à l'âge adulte à travers la série d'épreuves qui s'imposent à Poucet. Elle révèle ainsi, sous l'exemplarité du parcours méritant du plus petit et du plus jeune de la famille, capable de sauver ses frères, la valeur axiologique de la fable, rendue bien plus morale que dans le conte de Perrault.

8 Cependant, force est de constater qu'un tel traitement du conte-source est l'exception plutôt que la règle, l'essentiel du répertoire consistant en libres adaptations, voire en simples effets de citation du conte littéraire, comme dans En attendant le Petit Poucet (2001) de Philippe Dorin. Présente dès le titre et la première réplique de la pièce, la référence à Perrault disparaît ensuite presque totalement, à mesure que l'intrigue s'autonomise, tout en restant implicitement fondatrice pour l'ensemble de l'œuvre et en multipliant les emprunts : deux enfants abandonnés, Le Grand et La Petite, errent dans le vaste monde à la recherche « du petit coin où on pourra retirer [ses] chaussures et poser [ses] pieds sur un petit tapis ", accompagnés d'un petit caillou blanc, cependant qu'un chariot passe au loin, porteur d'autres cailloux blancs. Ce périple les conduit finalement, non pas à la réconciliation avec les parents, mais dans une maison où ils sont attendus par quelqu'un "qui cherche désespérément une histoire ", révélant que ce "petit coin», objet de la convoitise des enfants, n'est autre qu'une page blanche, métaphore transparente du conte en train de s'écrire.

Plusieurs stratégies auctoriales sont susceptibles d'être identifiées au sein d'un abondant répertoire de transpositions : d'abord, le séquençage de l'intrigue, conduisant à en isoler un épisode considéré comme scène primitive de la fable ; ensuite, le changement de point de vue, visant à donner la parole aux personnages considérés comme secondaires ou subalternes, voire franchement négatifs, quitte à requalifier le système axiologique ; enfin, la dilution du personnage éponyme, dont l'identité devient générique, ce qui s'exprime notamment par toutes sortes d'étymologies fantaisistes.

10 La désinvolture ostensible par rapport au conte littéraire est affirmée de façon récurrente dans la plupart des déclarations d'intention d'auteurs et de metteurs en scène désireux de déconstruire le conte. La Canadienne Claudine Galea se plaît à insérer subrepticement dans le récit cadre de Petite Poucet (2009), à propos de l'étonnante capacité d'affabulation de la jeune fille, «Le Petit Poucet a toujours été son conte préféré7 $»($ p. 66). C'est également 
le cas de Laurent Gutmann, affirmant dans Le Petit Poucet, ou Du Bienfait des balades en forêt pour l'éducation des enfants (2012) :

J'aborderai l'adaptation du Petit Poucet pour la scène non comme une digression, une rêverie, ou un commentaire, mais en souhaitant rester au plus près de sa narration, de l'évidence cauchemardesque du récit. Les terreurs auxquelles le Petit Poucet est confronté - celles de l'abandon, du meurtre, de la dévoration - seront ici les nôtres. Avec lui, nous retrouverons à la fin la maison de nos parents, à la fois changés et pourtant les mêmes ${ }^{8}$.

11 Cette curieuse et paradoxale invocation d'une certaine licence poétique et onirique, sous couvert de se livrer à un " commentaire ", conduit ici à la revendication d'une forme de subjectivation et d'appropriation du conte-source, cependant que le titre même révèle une ambition ironique par rapport aux finalités édifiantes et aux vertus pédagogiques supposées du conte de référence. C'est à partir de la même perplexité devant l'immoralité de cette « histoire d'un couple qui a abandonné ses enfants, une fois, deux fois, et qui n'arrive pas à se le pardonner » (p. 7) que le Béninois José Pliya met en évidence dans Mon Petit Poucet (2011), par le pronom possessif, la double démarche d'un père (« Le père que je suis a des enfants, qui [...] adorent qu'on leur raconte des histoires ») et d'un fils («Mon père me le racontait. Il était le bûcheron, j'étais le Petit Poucet»), tout en replaçant le conte dans la perspective d'un héritage mythologique plus large : «Quel monstre ou quel ogre faut-il être pour faire, froidement, en toute conscience, un tel choix? De quelle lignée antique - Abraham, le Dieu du Nouveau Testament, Agamemnon... - doit-on descendre pour survivre à ça ${ }^{9}$ ? »

Le même type de revendication auctoriale motive Gilles Granouillet, dans Poucet, pour les grands $(2012)^{10}$, à prendre le conte à revers : « Et si, pour une fois, le conte changeait ? Et si chacun prenait son destin en main?» Pour ce faire, il focalise sa transposition sur l'épisode emblématique de la rencontre avec les ogresses, donne la parole aux personnages considérés comme secondaires, se défend de toute imitation servile et met en abyme l'adaptation en convoquant sur scène le conte-source pris en tant que tel :

L'histoire que nous allons vous conter s'inspire de ce moment précis : la rencontre de Poucet avec les ogresses. Mais attention, nous n'allons pas plagier Charles Perrault! Dans notre histoire à nous, parmi toutes ces ogresses, il y aura une fille beaucoup plus maligne que les autres, une jeune ogresse qui se sent bien seule entre une mère qui fait l'autruche et une sœur complètement imbécile; une ogresse qui se pose beaucoup de questions sur son père, qui ne mange pas de viande et... qui aime les livres! Et justement, quand elle rencontre Poucet, elle a déjà lu « Le Petit Poucet »; elle connaît donc la fin de l'histoire; elle sait ce qui va lui arriver au milieu de la nuit!

Refusant à son œuvre la catégorie de "réécriture ", "puisqu'elle ne suit pas sa trame narratrice ", et à sa pièce celle de " conte théâtralisé », il s'affranchit de la dimension patrimoniale de ce conte "monument» et conteste l'occultation de la violence de la situation dramatique, envisagée " du côté des filles »: il y voit le ferment d'une forme « de résistance et de résilience ${ }^{11}$ ». C'était déjà le parti-pris de Pierrette Fleutiaux et Monic Cecconi-Botella dans La femme de l'ogre (1989), "opéra-conte » en huis-clos entre l'Ogre, " grand prédateur, semant la terreur et la peur, pourtant capable d'amour, les Ogrelettes (les filles de l'Ogre), des enfants inconscientes du drame qui se joue devant elles et avec elles, joyeuses, cruelles jusqu'à la mort, la Femme de l'Ogre, tendre, fragile, humaine ${ }^{12}$ ". Poucet, complètement éclipsé, est dès lors réduit à la simple fonction d'embrayeur d'un drame à la fois sentimental et sanglant où l'Ogre et les Ogrelettes, à la recherche de chair fraîche, finissent par s'entre-dévorer. 

caillou dans la botte $(2011)^{13}$, en la personne de Nikolaï Ogrovsky, qui «pour la première fois, prend la parole publiquement " pour " raconter son histoire, dire sa vérité ", à la manière d'une enquête policière ou d'un procès criminel. Ce spectacle d'ombres mêlé de marionnettes, de maquettes, d'objets, «change le point de vue initial du conte et adopte, pour une fois, le regard de l'ogre, ainsi qu'un peu de sa mauvaise foi ». Autrement dit, l'Ogre est présenté sous la forme d'un "brave carnivore, certes légèrement cannibale ", «volé, dupé, ruiné, et ce en une seule nuit, par un misérable rejeton ». Dès la scène d'ouverture, le récitant, sous les traits duquel transparait l'ogre, prétend apporter un démenti public aux allégations frauduleuses du conte et en propose le travestissement burlesque :

Vous connaissez le petit Poucet? Le génie qui sauve ses six frangins, les parents tellement pauvres, les petits cailloux blancs, la forêt, la nuit, la peur, le vilain ogre et ses terribles dents, vous connaissez?

On vous ment. Je m'appelle Nikolaï Ogrovsky. Je suis un carnivore, un viandard, un croqueur de marmots, un ogre. Je suis l'ogre. J'ai été trompé, maltraité. On m'a tout pris - ce que j'avais, ce que je suis, tout. On raconte n'importe quoi, mais je vais vous dire la vérité.

Tout a commencé parce qu'à l'autre bout du pays, un type miteux s'est pris les pieds dans sa propre vie. Ce type était bûcheron, mais plus personne ne voulait de son bois $^{14}$.

Le récitant intervient ensuite de façon récurrente en appui de l'action des parents, qu'il compare à « des vraies crapules, des vauriens, des moi-moi égoïstes » (p. 10). Il en propose un commentaire décalé, tout en filant la métaphore de la dévoration : «Pensez de moi ce qui vous chante, mais le mensonge, jamais. Faire des choses pareilles à ses propres enfants, la chair de sa chair, le sang de son sang ! Le sang... » (p. 8)

La dilution du personnel dramatique peut en outre se conjuguer au brouillage des castes, des générations et des genres, comme dans Petite Poucet de Claudine Galea, " pièce pour acteurs mais aussi, selon les options de mise en scène, voix, marionnettes, cinéma et chorégraphie » (p.33). La féminisation du personnage éponyme, surnommée Violette et jugée " absolument ravissante ", flanquée de ses sept sœurs aux noms de fleurs, aux prises avec le Docteur Logre, "très séduisant ", met au premier plan les rapports de séduction, tout en exacerbant les pulsions possessives de la Mère à l'aide d'une langue à la fois argotique et poétique :

Ma petite mon poussin mon ouistiti mon colibri ma mignonnette ma linotte ma petiote ma première ma dernière mon mini-cœur ma petite fleur mon Poucet mon Petit Poucet

J'ai un bébé

Je n'en ai qu'un

Il est mon mien mon tout mon bien

Mon petit mon bébé mon poussin mon Poucet

[...]

Te garderai aussi longtemps que je pourrai

[...]

Si je pouvais t'éterniser l'amour serait l'amour serait tétanisé

Mon unique mon Poucinet

Ma toute seule

Ma toute mienne mon tout mon bien mon lien mon rien

(Dix, p. 60) 
17 Brouillant les pistes tout en multipliant les emprunts, les transpositions théâtrales procèdent ainsi au détournement référentiel du conte littéraire afin de donner au personnage une vocation paradigmatique.

\section{Génériser la figure du personnage éponyme}

Plus que par la multiplication plurivoque de points de vue et de systèmes énonciatifs, avec pour horizon d'attente la réhabilitation de personnages secondaires (et à plus forte raison négatifs), c'est par la généricité du personnage de Poucet que s'exprime le travestissement burlesque du conte-source dans de nombreuses transpositions. C'est ainsi que le personnage éponyme est affublé de toute sortes de dénominations régressives, étayées sur des étymologies fantaisistes ou des jeux onomastiques gratuits. Chez Philippe Dorin, dans En attendant le Petit Poucet (2001), la scène d'exposition s'ouvre sur un jeu de mots programmatique pour l'ensemble de la pièce, exprimé par Le Grand :

L'histoire du petit poucet! L'histoire du petit pouc'est... l'histoire du petit pou! L'histoire du petit poucet, c'est l'histoire du petit pou. Il en avait plein la tête. Et ça le grattait, ça le grattait. Et chaque fois qu'il se grattait la tête, pour pas qu'on voie que c'était à cause des poux, il disait : «J'ai une idée ! ». (Scène 1, p. 9)

L'exercice formel de cet ouvroir de littérature dramatique potentielle motive la métaphore générée au moyen de l'assonance équivoque en transformant le handicap, ou plus précisément ici le stigmate social du "pouilleux», en avantage comparatif: il métamorphose ainsi Poucet en une sorte de clochard céleste, curieusement crédité du prestige de ce qui devrait pourtant être la cause de sa disgrâce. Le procédé d'habilitation paradoxale du personnage par son seul mérite personnel est encore plus flagrant chez Pliya dans Mon Petit Poucet, dont le bûcheron Guillaume reprend à son compte la métaphore du pou en évoquant son fils en ces termes :

Il est maigre, malingre, un petit peu niais ou nigaud, en tout cas il ne parle presque pas. [...] Quand on parle on pourrait croire qu'il écoute, mais non : c'est une tête de linotte, une tête en l'air, un peu demeuré, un peu tebê ou débile léger. Il sourit tout le temps : le sourire idiot, le sourire neuneu d'un gamin qui n'est franchement pas beau. Il ne sait rien, il ne fait rien. Dans une maison, un inutile qui ne sert à rien, on appelle ça un pou, un parasite. Voilà pourquoi à sa naissance je l'ai surnommé «mon Pou, mon petit Pou». Ses frères eux, l'appellent tendrement «sale petit Pou!». Rien de méchant! Il faut bien rire de temps en temps. (p. 19)

Révélant toute la violence symbolique de l'appellation diffamatoire, sous couvert de diminutif affectueux, le qualificatif de "petit Pou » relève la stigmatisation dont il est victime à l'intérieur même du cercle familial. Elle contraste avec la démesure de la légende écrasante des pères, auréolés de prestige, quelques pages plus loin : «Un combat de titan qui reste dans les mémoires. On en parle encore dans les veillées... Une légende mon père, un héros. (Temps) Comme lui, je suis devenu bûcheron, père d'une famille nombreuse [...].» (p. 21) Mais c'est finalement dans la dynastie hétéroclite des héros de petite taille que s'inscrit le Pou à l'issue de son parcours initiatique: «[...] prenez les contes, ils prétendent que mon fils est l'égal de Dédale, de Thésée, de David ou de Kirikou... Alors moi, je voulais savoir la vérité. Je voulais connaitre la véritable histoire de mon fils. J'ai fermé les yeux. Je les ai ouverts. » (p. 41)

21 Caroline Baratoux, dans Le Petit Poucet, propose encore une autre hypothèse onomastique tout aussi délirante, associant d'emblée le personnage du Poucet à la voracité. Elle explicite ainsi la nécessité de surmonter le stade oral (Bettelheim, 1999 : 243) et annonce 
le fantasme de dévoration qui structure le conte et s'organise autour de son incomplétude :

Je suis le fils unique d'une famille de sept garçons.

Ils sont tous arrivés deux par deux, mes frères.

Maman poussait des cris stridents à chaque fois.

Avec moi ce fut différent, je veux dire que maman,

cette nuit-là, dormait profondément quand j'ouvris les yeux.

C'est la première fois que je l'ai regardée,

elle souriait dans son sommeil silencieux.

J'avais faim ; alors, de mon pouce,

j'ai pris son sein,

et puis le lait qui coulait...

Peut-être que c'est pour cela qu'on m'appelle

le Petit Poucet

et non parce que je suis de petite taille, grand comme le pouce de mon père,

qui lui est grand et fort

car il est bûcheron.

(Scène 1, p. 7)

Sans identité marquée, Poucet devient dès lors, dans une proportion non négligeable du théâtre contemporain, un pur et simple réflecteur du système des personnages et surtout, des rapports de forces qu'ils expriment : il ne prend sens qu'en continuité ou en rupture avec une généalogie qui le dépasse et cherche à l'accabler. L'héroïsation paradoxale et édifiante qui lui était traditionnellement associée est donc délibérément compromise ou pour le moins, déplacée, remotivant ainsi la portée symbolique du conte.

\section{Réamorcer la charge mythologique}

Poucet n'est pas le seul personnage à faire l'objet d'une captation d'attributs: les caractéristiques de l'Ogre sont également régulièrement diffractées et ses caractéristiques mythologiques, tout comme ses travers anthropophages, contaminent l'ensemble des personnages, mettant au premier plan le motif de la dévoration qui constitue une des obsessions du théâtre contemporain. C'est ainsi en pleine crise de couple entre Marie-Léonie Logre et son mari Poucet que Jean-Claude Grumberg, dans Mange ta main. Conte pour enfants précoces et adultes attardés (2006), construit l'intrigue à partir d'une métaphore filée : un dense réseau d'expressions telles que «se mordre les doigts » ou "manger sa main » configurent un substrat imaginaire d'une particulière efficacité dramaturgique. Dans le Petit Poucet de Caroline Baratoux, qui représente pourtant la version récente la plus fidèle à la trame narrative du conte-source, Poucet fait l'expérience tragi-comique d'une dilution des attributs de l'ogre sur sa descendance. D'emblée l'Ogresse, après avoir dit de son mari qu'il « n'est pas tout à fait une personne ", puisqu' « il a la tête dans le ventre / et le ventre dans la tête ", et que sa maison « est un cercle dont on ne sort jamais... », car "c'est encore la forêt à l'intérieur ", définit ses propres filles comme "des têtes sans bouches... des filles bouchées...", qui ne parlent jamais et ne cessent des claquer des dents (scène 12, p. 28-30). Cette mise en garde explicite n'empêche pas Poucet, moins roué qu'à son habitude, de se faire surprendre, lorsque la nuit venue, il s'approche du lit des filles de l'Ogre et fait tout à la fois l'expérience troublante de l'altérité et de la similarité. D'abord, «il regarde intensément la fille la plus proche", puis, lorsqu'elle se réveille, "ils se regardent intensément », ce qui donnerait à penser que quelque chose de l'ordre de l'émotion se noue entre eux. Mais la 
jeune ogresse met brutalement fin à cette hypothèse d'une idylle naissante entre ennemis en affirmant dans un rire : " Père te mangera... et je te sucerai les os... » (scène 15, p. 35). Poucet tranche finalement l'ambiguïté et refuse de renchérir sur cette pulsion meurtrière, sans pour autant renier l'émoi sensuel qui l'a d'abord animé au contact de cette silhouette cadavérique :

Je n'avais jamais vu de filles jusqu'à aujourd'hui...

C'est joli, une fille... Les cheveux sont plus longs que les miens...

Et ils sont si rouges...

Et cette peau!

Elle est transparente...

(Rêveur) [...]

C'est joli, une fille... mais différent... spécial...

Pardon, mais nous on s'en va.

(Scène 16, p. 37)

C'est également au cœur d'un drame sur l'exclusion d'une petite fille boulimique dans la cour de récréation que Nathalie Papin décide de placer l'amplification métaphorique de la dévoration, prémices d'une fable métaphysique dans Mange-moi (2002). Le ton est donné dès l'évocation de la compulsion gloutonne d'Alia, qui refuse de partager son goûter: «Parce que si je n'ai pas le ventre plein, j'ai l'impression que je vais m'envoler, très haut, plus loin que les étoiles, et les galaxies. Il paraît que dans l'univers il y a des trous noirs. J'ai peur de tomber dedans si je décolle. Alors je mange pour être lourde. J'ai mal quand j'ai faim. Je préfère mourir que d'avoir faim. » Aussi, lorsque la « grosse » décide de fuir la violence ordinaire de ses camarades de classe, un dictionnaire sous le bras, c'est à un Ogre squelettique et anorexique qu'elle se heurte : il a décidé d'arrêter d'engloutir les enfants et de tromper sa faim en ingérant l'horizon, surgi de nulle part, dans un grand bruit de mastication. Il est «devenu transparent» (p. 22) à force de maigrir en régurgitant les enfants qu'il dévorait, si bien qu'il a été jugé dangereux par le "tribunal des ogres" (p. 23) et « expulsé de la cité des ogres parce qu'[il] recrachai[t] les enfants et les renvoyai [t] chez eux» (p. 28), après avoir été marginalisé pendant toute son enfance: «[J]'étais tellement gringalet que les autres me bousculaient et me croquaient dedans. » (p. 23) Nul doute que ces personnages à contre-emploi ( Tu parles comme les ogres. D'ailleurs tu ressembles à une ogrillonne, une petite ogresse ", p. 19) puissent tomber amoureux l'un de l'autre, donnant littéralement chair à l'Ogre-squelette et redessinant la silhouette de la jeune fille soudain complexée par le désir. Dans l'intervalle, l'Ogre se reconvertit en lit de fortune (« Mon estomac est devenu une chambre à coucher », p. 17), soulevant ses côtes pour prêter refuge à la jeune fille, refuse l'injonction de la manger qui fait le titre de la pièce et déclare son trouble de l'identité ( "Je suis ogre et pas ogre », p. 18) ; quant à Alia, elle s'aventure sur la ligne d'horizon, à la rencontre d'un mangeur de mémoire, d'une dévoreuse de temps et d'une dévoreuse de livres, afin de le sauver en lui offrant finalement la rédemption.

Si la pièce se plaît à déconstruire l'enchantement, en ironisant sur les attributions de l'ogre et sur l'horizon d'attente du conte (« je D-O-I-S manger les enfants parce que je suis un ogre, toi, petite fille, tu D-O-I-S avoir peur des ogres », p. 19), c'est pour aussitôt en proposer un univers de substitution onirique qui prend sens dans le cadre du voyage initiatique et de la quête métaphysique d'Alia au sein du «monde des dévorants » (p. 44). Elle y est confrontée à des figures allégoriques, récoltant des attributs magiques (extrait de chagrin pur qui extériorise la mélancolie, larme artificielle qui suspend le temps qui passe, livre blanc qui modifie le cours de l'histoire). Elle lutte donc, par son action, contre 
le double destin contrarié des personnages : à peine mis en cause, l'univers du conte merveilleux est donc immédiatement remotivé et réinvesti par l'amplification métaphorique de la dévoration, qui prend rapidement les allures d'une véritable parabole, alors que le langage lui-même se délite, voire se décompose : « [I]l est a-n-o-r-e$\mathrm{x}-\mathrm{i}-\mathrm{q}-\mathrm{u}-\mathrm{e} »(\mathrm{p} .40)$.

Entièrement construite autour de la dialectique entre dévoration et régurgitation, entre faim hyperbolique et impossible digestion, entre appropriation et restitution, la pièce offre ainsi une sorte de variation à thème constant. Force centripète, l'ogre apparait rapidement comme une sorte de cyclone absorbant le monde pour mieux le recracher ailleurs : «C'est mon repas d'hier soir : un désert, une nuit étoilée, et les lignes d'horizon que je garde, ça peut toujours servir. Demain matin, j'irai en Chine gober un lever de soleil. Tu l'auras pour toi toute seule. » (p. 30) Son appétit sans limite entraîne un tropplein ontologique qui, dans un paradoxe apparent, génère en lui un puissant vide métaphysique : " Il y a encore des planètes, des galaxies. On n'a jamais fini. On peut aller jusqu'à néant. »(p.37) C'est donc tout naturellement qu'il en arrive à préconiser l'autophagie: "Il m'est impossible de ne pas engloutir quelque chose, aussi, je me mangerai moi-même, un tout petit peu chaque jour. [...] L'ogre commence à se manger le doigt. C'est assez bon! Les Ogres devraient se goûter eux-mêmes avant de se gaver des autres.» (p.45) Une telle autodestruction contamine le dialogue théâtral. Ainsi, lorsqu'Alia rencontre le Bonhomme, personnage muni d'un "ventre avec un énorme œeil dessus, au milieu, fermé » (p. 61), ce personnage-cyclope ou ce phare d'un genre très spécial lui explique son attribution. Elle consiste à dévorer des yeux les nageurs perdus dans l'horizon marin: "Quand ils sont très près, je me retourne, j'ouvre mon œil, et mon regard les avale. J'en éprouve un grand plaisir, jusqu'au prochain. [...] Bien voir c'est dévorer des yeux. » (p. 62-63) Tous les personnages, sans exception, ont donc quelqu'un ou quelque chose à dévorer, depuis le mangeur de mémoire jusqu'à la dévoreuse de livres. Et tous ont une justification à fournir: «Je ne vole pas, je débarrasse. [...] C'est une trouvaille que j'ai faite pour devenir éternel: me nourrir de la mémoire des autres » (p. 50) ; « Je ne les mange pas, je les dévore des yeux. C'est pas pareil. Les gens aiment bien qu'on les dévore des yeux » (p. 66); «Il n'y a rien de meilleur au monde. Je dévore les livres depuis que j'ai compris que c'était de la chair. [...] Seulement, depuis ce jour, je n'ai jamais pu revenir dans le monde ordinaire » (p.68). Pulsion universelle, la dévoration organise par conséquent et prend en charge le récit, se propageant à l'ensemble des personnages, tout en réactivant la charge mythogène et la portée symbolique du conte merveilleux.

L'auteure canadienne Suzanne Lebeau suit une démarche comparable avec son adaptation de L'Ogrelet (1997) ${ }^{15}$. Elle campe d'emblée un décor de pure convention sur lequel ironisent allègrement des didascalies. Elles font appel à la compétence culturelle du lecteur : « Une maison, dans un bois, comme on les imagine dans les contes, frustre, inconfortable. » Elles ont en outre pour seule fonction de suggérer un univers sensoriel évocateur, sans aucun illusionnisme scénographique: "Une école de village dont on parle souvent. Elle peut être au loin dans une forme miniaturisée. Dans cette école, des ombres et des sons." Elles revêtent enfin une dimension symbolique : "Une cabane de chasseurs abandonnée et la forêt comme un étau, tout autour.» (p.12) La pièce prend le destin des personnages à revers et superpose plusieurs figures, en particulier l'Ogre et le Petit Poucet, présenté comme un ogre en puissance, voire en devenir. Elle évacue en apparence la figure de Poucet ou plutôt, l'agrège à celle de l'Ogre, devenu le petit Simon de six ans, qui a «le regard d'un 
adulte fou dans son visage d'enfant sage » (p. 34). Celui-ci fait à l'école l'expérience de pulsions meurtrières à la seule vue de la couleur rouge. Il découvre, par la confidence de sa mère, qu'il est le fils d'un Ogre, lui-même fils d'une dynastie d'ogres depuis plusieurs générations, mais qui a décidé de conjurer le sort et de l'abandonner pour le soustraire à la menace de l'infanticide. Le jeune garçon s'impose alors un exil initiatique dans une forêt profonde, constitué de trois épreuves révélées par son père dans une lettre testamentaire avant sa disparition: il doit d'abord se soumettre à la tentation de cohabiter sans le dévorer avec un coq pendant une journée, simplement pourvu de fruits, de légumes et de quelques cruches d'eau. Il doit ensuite réitérer la même expérience pendant une semaine avec un loup, et enfin pendant un cycle de lune avec une petite fille (p. 44-45).

De telles épreuves sont destinées à le guérir de son "ogreté » et à lui permettre de surmonter ses fantasmes de dévoration et son attirance vampirique pour «l'odeur du sang » (p. 25) et pour « le goût du sang» (p. 33). Il accepte donc le principe d'abnégation et de contrôle des pulsions, sans pour autant renoncer à sa part de "sauvagerie ", réconciliant en lui «le loup, / qui s'est inscrit dans notre mémoire comme l'ennemi », et renouant avec notre "terrible hérédité » (poème postface, p. 81). Fable humaniste et anthropologique sur le fragile équilibre entre Nature et Culture, la pièce de Suzanne Lebeau met en scène l'éveil d'une conscience à sa généalogie monstrueuse (" Où l'Ogrelet apprend qui est son père et le sang qui coule dans ses veines moitié-moitié », p. 39) et l'expérimentation de sa propre altérité menaçante («Où l'Ogrelet reconnaît le danger avec lequel il doit apprendre à vivre », p. 59) : «Je suis le fils d'un Ogre qui a mangé ses filles !» (p. 43) S'imposant à lui-même le parcours initiatique de la tentation surmontée, il apprend à vivre avec ses pulsions de meurtre et à raisonner son instinct morbide et sexuel de prédation; plus exactement, il apprend à le sublimer, avec l'aide de sa compagne Paméla, comme le révèle malicieusement la pointe finale, alors que Simon s'apprête à rencontrer son père, revenu lui-même de sept ans d'épreuve initiatique :

Pour occuper nos mains et nos esprits, Paméla et moi, on modelait des petits orteils avec la cire des chandelles. [...] L'orteil de Paméla, le vrai, je l'ai croqué, mais je ne l'ai pas avalé. Je le garde dans ma poche avec la petite goutte de sang séché qui ne veut pas partir. (p. 76)

29 Trophée fétichisé, l'orteil de la jeune fille agit comme un talisman érotique censé rappeler le jeune ogrelet à lui-même, tout en déroulant la fable politique : il reprend à son compte la métaphore hobbesienne de l'homme-loup et offre les conditions de possibilité d'une «trêve avec soi-même et avec le monde » (p. 81).

Ces pièces de la dévoration revitalisent ainsi un substrat mythologique tantôt de façon explicite, comme lorsque José Pliya reproduit dans l'édition et évoque dans les didascalies de Mon Petit Poucet des tableaux tels que Saturne dévorant un de ses enfants de Goya ou David avec la tête de Goliath du Caravage, tantôt de façon plus implicite. Elles remotivent un imaginaire symbolique inspiré par un processus de métaphore filée ou d'amplification métaphorique: «Car si L'Ogrelet est un conte moderne, / Il puise dans la tradition ses métaphores. » (p. 81)

\section{Actualiser et déterritorialiser la fable contrariée}

Loin de se réduire au recyclage d'un matériau mythologique et symbolique, la transposition est souvent doublée d'une forme d'actualisation et de détournement, 
comme chez Laurent Gutmann, écrivant avec son Petit Poucet « une version pour la scène et pour aujourd'hui », ou chez Fabrice Murgia dans Le Chagrin des ogres $(2010)^{16}$, librement inspiré par des faits divers ainsi que par le blog de Bastien Bosse, étudiant allemand qui s'est donné la mort après avoir ouvert le feu sur ses camarades dans son ancien lycée, et par des entretiens avec Natascha Kampusch, jeune Autrichienne enlevée et séquestrée pendant plus de dix ans.

Le Chagrin des ogres, c'est le récit d'une journée au cours de laquelle des enfants vont cesser d'être des enfants. [...] Leur tentative de fuir dans un imaginaire formaté par nos nouvelles manières de communiquer. Le désespoir de ceux qui hurlent à l'aide, sans que l'on sache réellement lesquels d'entre eux détiennent des rêves et des bombes pour se venger de ceux qui ne les entendent pas.

Le Chagrin des Ogres, c'est notre façon d'enterrer notre enfance.

Si la relation à Perrault est de prime abord relativement ténue, elle porte cependant la réflexion sur le malaise d'une génération, envisagé à partir de « testaments d'enfants » et de faits divers de school shootings, dans un spectacle documentaire mêlant étroitement performance et projection vidéo d'images d'archives ou de captation directe pendant la représentation. Partie intégrante du dispositif spectaculaire ( La vidéo, c’est de la lumière qui bouge »), l'image projetée dissémine des effets de réel dans un univers globalement symbolique, tout en subvertissant une société de l'image entrée dans l'ère des nouveaux médias. Le spectacle vise à susciter la sidération du public au moyen de l'« accroche au réel, la plus crue possible, pour après créer un envol plus onirique ». "Cette fable onirique doit transpirer le vécu", affirme l'auteur et metteur en scène, avant de confier que son spectacle, en configurant un « espace mental ", «s'adresse à la part d'enfance qui s'est retranchée derrière notre cerveau, étouffée par les règles qui conditionnent notre comportement adulte et responsable»: elle cherche à "toucher derrière le cerveau " une jeunesse caractérisée par son «incapacité à se révolter » (Murgia/Delhalle, 2009).

La stratégie de réarticulation entre poétique et politique est assez similaire dans le " conte à mourir debout»( (p.27) dont relève Mon Petit Poucet de José Pliya, qui à l'actualisation ajoute une forme de déterritorialisation, déplaçant la fable en Afrique, là où les notions de famine et de désolation prennent un sens concret et immédiat. C'est ainsi que la situation de départ détaille longuement la « famine sans précédent » qui «a sévi dans le village et alentour. Une famine comme on n'en a jamais connue» (p. 22). Renouant avec la tradition des contes de griots, plaisamment qualifiés de "fariboles " ( Les contes racontent des fariboles », p. 37), c'est cette fois sur la puissance d'évocation du récit plutôt que sur le choc des images que repose la pièce de l'auteur béninois installé aux Antilles. Il renverse la situation de départ du conte-source, puisque dans cette pièce, c'est un père pris de remords qui se lance à la recherche de son fils et entreprend un long et périlleux voyage initiatique destiné à lui faire réapprendre à être père, afin de retrouver une autorité perdue. Il accomplit un parcours à la fois historique dans une Afrique désolée par la guerre et symbolique autour de la terre. Le drame de la culpabilité et de l'abandon tourne ainsi à l'évocation d'un « pays-chaos » sinistré, d'une société à la dérive où les enfants paient un lourd tribut à la folie meurtrière des hommes :

Et je suis parti dans le vaste monde à la recherche de mon fils. J'ai vu un pays-chaos dévasté par la famine, où il n'y avait plus rien à manger, rien pour les enfants. J'ai vu des campagnes en feu, des campagnes en flammes livrées aux pilleurs de ventre, aux violeurs de gosses. J'ai vu des cités en ruines, des cités millénaires où jadis les dromadaires paissaient. Elles étaient tenues par des soldats, des enfants-soldats. J'ai vu des villes-carcasses, des villages-poubelles, des hameaux-décharges, des comtés- 
déglingues et des territoires entiers où l'alliance des loups et des chiens avait eu raison de l'homme. J'ai vu des hommes démontés, des femmes saccagées, des fillettes qui n'ont plus de bras que l'on retrouve à Calcutta, des garçons projectiles qui se jettent dans le Zambèze. J'ai vu toute cette misère et toute cette désolation, et je n'ai pas trouvé mon fils. (p. 38) bûcheron rencontre des êtres à l'agonie (interprétés par la même actrice que celle qui joue la Bonne Femme) : tous prétendent avoir vu son fils et tous lui font un récit différent de son affrontement mythique avec Orcus. C'est d'abord en pays occitan un garçon mourant qui prétend l'avoir connu, puis en pays aborigène une fillette se vidant de son sang qui affirme l'avoir rencontré, et lorsque finalement, de guerre lasse, Guillaume revient chez lui, son fils l'attend, rentré aguerri de son périple. Mais ce n'est que pour mieux repartir, sur la pointe des pieds, dès que s'endort son père... La clôture narrative et la structure itérative du récit, qui s'achève presque sur les mêmes mots que la formule de départ, conformément aux traditions conteuses, laisse penser que Poucet s'apprête à partir de nouveau. Elle déréalise le cadre référentiel que le récit s'est pourtant évertué à évoquer dans une langue argotique forte et inventive qui, loin d'euphémiser la violence, cherche à l'exhiber et à lui donner toute sa puissance, sans pour autant renoncer à l'humour. Fidèle à l'esprit du conte, Pliya, en le déplaçant dans une Afrique dévastée qui en remotive la signification, en offre ainsi une actualisation saisissante, voire sidérante qui suscite un certain effroi.

\section{Libérer le récit, matérialiser l'imaginaire}

Certaines options dramaturgiques constituent une gageure pour la mise en scène contemporaine et nécessitent un dispositif scénographique particulier, comme dans Mange-moi de Nathalie Papin, où il faut mettre en scène la dévoration, mais aussi l'engloutissement de la ligne d'horizon ou la figuration de personnages allégoriques... Dans un tel contexte, il est tout sauf fortuit qu'un certain nombre de dramaturges aient à cœur de monter eux-mêmes leurs propres contes théâtralisés (Pliya, Gutmann, Granouillet, Pommerat, Py), ou pour le moins de collaborer activement à leur mise en spectacle, comme Suzanne Lebeau au Caroussel ou Caroline Baratoux avec l'atelier HorsChamp. Si un certain nombre de mises en scène trouvent des solutions pratiques parfois astucieuses afin de convoquer sur scène l'enchantement, d'autres placent ces impératifs techniques au centre de la dramaturgie.

L'un des enjeux des transpositions dramatiques du « Petit Poucet » consiste, en libérant le récit des contraintes formelles du conte littéraire, à articuler la dramaturgie à une scénographie adéquate: elle vise à transcrire un univers poétique, allégorique, métaphorique, onirique, en imaginaire corporel, visuel et sonore. Contrainte surmontée, la matérialisation de l'imaginaire du conte reconfigure donc l'espace du plateau. Parfois, la mise en scène se contente de simples effets de suggestion au service de la narration, comme chez Pliya dans sa propre mise en scène de Mon Petit Poucet au Centre dramatique de la communauté française Wallonie-Bruxelles en 2011: le Père (Dieudonné Kabongo) évolue dans la pénombre, sous un plafond dont la hauteur varie, et la Bonne Femme (Sophia Leboutte) apparaît ponctuellement dans l'encadrement d'une fenêtre luminescente. Parfois, le spectacle procède par simple citation visuelle, comme dans Poucet pour les grands de Gilles Granouillet, où la compagnie Travelling Théâtre projette 
sur la scène de Saint-Étienne en 2012 les célèbres gravures de Gustave Doré illustrant le conte de Perrault, tout en créant en direct une musique de scène destinée, à la manière du cinéma, à souligner l'action et ses effets dramatiques. Parfois, comme dans Un Caillou dans la botte de Simon Grangeat, créé par Clément Arnaud et la compagnie Traversant 3 à la MJC Jean Macé de Lyon en octobre 2011, les personnages apparaissent sous forme d'ombres (soixante-quinze marionnettes, formes transparentes imprimées de 5 à 30 centimètres et tenues sur des baguettes de bois) par rétroprojection panoramique en gros plan sur le plateau, où deux plasticiens-marionnettistes projettent espace et personnages sur un cyclo central placé dans le lointain, pendant qu'un musicien crée une "véritable bande-son cinématographique", mêlant guitare, clavier et matériel de déformation et de traitement d'effets sonores. Parfois, ce sont de simples dispositifs scénographiques qui cadrent l'espace et servent de point d'appui à l'expansion de l'imaginaire, comme avec les sept voiles tour à tour transparents et opaques de la mise en scène du Petit Poucet de Laurent Gutmann au Théâtre des Cinq Diamants en 2011, évoluant selon l'éclairage et brouillant les contours, de sorte que « l'expérience du Petit Poucet soit aussi la nôtre, qu'avec lui nous perdions nos repères ${ }^{17}$ "; ou comme avec le système de cordes encadrant un espace polyvalent conçu par Pascale Nandillon, conseillée par Joël Pommerat, pour la mise en scène du Petit Poucet de Caroline Bartoux dans le cadre de l'Atelier Hors-Champs en 2008-2009:

L'aire de jeu est délimitée au moyen de plusieurs cordes, certaines rouges, tendues du sol au plafond. Voilà les piliers de la maison, voilà les arbres de la forêt, voilà des cordes vocales, une bouche, des entrailles. L'excès d'arabesques et de couleurs et la lumière font vibrer les verticales. Un petit arbre aux branches rouges sang, buisson ardent ou foyer des parents de Poucet et de l'ogre les abrite tour à tour ${ }^{18}$.

La plupart du temps, qu'il se manifeste par l'épure du plateau nu ou au contraire par un dispositif scénographique complexe, le spectacle joue sur l'espace transitionnel du conte en brouillant les référents spatiaux et temporels trop explicites (la masure des bûcherons, la forêt profonde, la terrifiante maison de l'Ogre), créant un univers onirique évocateur à partir de systèmes d'analogies ou de signalisation. Il en est de même dans le choix des costumes, comme on le voit par exemple à l'aide des costumes « réversibles » imaginés par Pascale Nandillon :

Si les costumes prennent en charge l'identité des personnages, ils peuvent à tout moment se retourner contre eux. Les acteurs pourraient être habillés d'étoffes réversibles: d'un côté, des teintes similaires à la forêt; de l'autre, du rouge, des entrailles. Les tissus des costumes pourraient se confondre aux motifs végétaux des tentures. (p. 10)

Mais c'est surtout l'univers sonore qui, comme chez Pommerat dans Cendrillon ou dans Le Petit Chaperon rouge, sculpte littéralement l'espace en dessinant un univers composé du souffle du vent dans les feuilles, du chant des oiseaux dans le ciel, des coups de hache au loin dans la forêt ou du gargouillis du ventre qui a faim, cependant que les comédiens s'adonnent à une sorte de ritournelle, «activité corporelle de chantonnement, un peu ritualisée, marqué par la simplicité et par la répétition » qui « désigne le moment d'une appropriation de son corps » (p. 11)...

Parti-pris interprétatif, la mise en scène de l'enchantement peut également faire retour au niveau de la dramaturgie au sein des nouvelles écritures scéniques, voire de façon plus large du théâtre contemporain, et par conséquent constituer la scénographie en principe organisateur de l'écriture. Si comme on l'a vu la plupart des attributs traditionnels du conte sont évacués des transpositions, les petits cailloux blancs font exception à la règle 
et bénéficient d'un traitement spécifique. Ils sont placés au cœur de la scénographie et même, de la dramaturgie de plateau d'un certain nombre de transpositions du conte.

C'est ainsi que Philippe Dorin, tout en plaçant son œuvre sous le patronage poétique de Samuel Beckett et d'Arthur Rimbaud, «Petit Poucet rêveur ", égrenant dans sa course, des rimes (citation liminaire placée en exergue de la pièce), organise En attendant le Petit Poucet à partir de la matière plastique éminemment scénique des petits cailloux blancs. Jeu formel sur la langue d'inspiration surréaliste, la pièce a de prime abord les allures d'une fable métaphysique contrariée où la quête mystique conduit résolument à la mort :

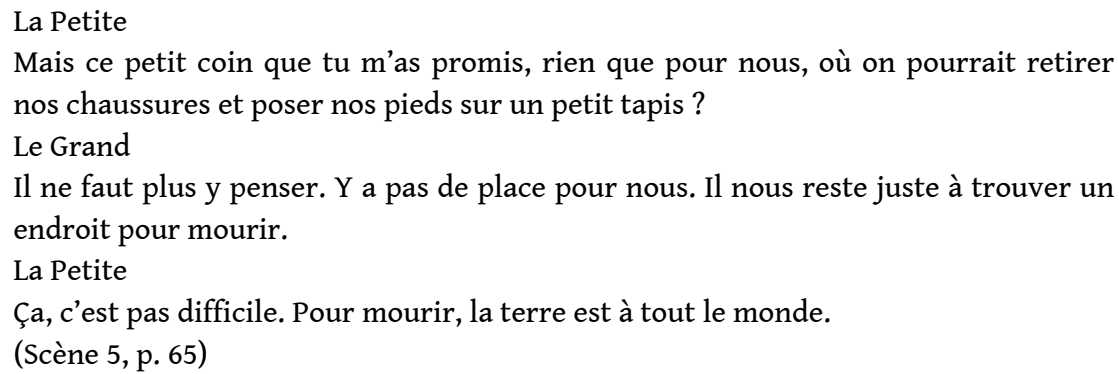

41 Cependant, c'est bien la dissémination du caillou personnifié qui organise le récit. L'objet sert en effet d'embrayeur de l'action, dans une scène d'ouverture où les enfants sont occupés à semer des cailloux (p. 9-14). Il est ensuite élevé au rang de personnage à part entière (« Le petit caillou blanc apparaît. Il rejoint La Petite. », p. 14, « Le petit caillou passe en faisant des petits bonds aussi », p. 41), puisqu'un petit caillou, qui suit Le Grand et La Petite pendant leur pérégrination, se voit désigné («Il nous suit depuis le début », p. 42), voire apostrophé («T'as entendu? Il faut que tu rentres.», p. 43). Et lorsqu'il n'est pas directement présent sur scène, le caillou personnifié est immédiatement invoqué par les personnages : «C'est un caillou comme un autre. $Y$ en a partout au bord des chemins. Tu ne vas pas t'apitoyer sur chaque caillou que tu rencontres!» (p. 44-45) Il donne lieu à des équivoques plaisantes, comme lorsque Le Grand demande son nom à La Petite qui lui répond qu'elle s'appelle Pierre :

Le Grand - Ce sont les garçons qui s'appellent Pierre.

La Petite - Non! Pour un garçon, on dit caillou. (p. 17-18)

L'évocation du caillou organise le séquençage de l'intrigue, par son intervention récurrente en ouverture de chaque scène, comme l'indique l'évolution des didascalies inaugurant chaque nouvelle scène : «Le petit chariot passe au loin, chargé de petits cailloux blancs» (p. 23, avant la scène 3) ; «Le petit chariot passe au loin, chargé de petits cailloux blancs. Un petit caillou tombe du chargement. » (p. 61, avant la scène 5)... Elle donne surtout sens à la quête symbolique, comme lorsque les deux enfants perdus retrouvent enfin le petit caillou qu'ils avaient congédié :

Réfléchis un peu! S'il était revenu, il serait arrivé derrière nous. [...] Ça veut dire que c'est nous qui l'avons rejoint. [...] Ça veut dire qu'on a fait le tour du monde. [...] Et qu'on n'a rien trouvé. [...] Y a pas de place pour nous. Il nous reste juste à trouver un endroit pour mourir.

(Scène 5, p. 64-65)

43 Le caillou clôt finalement la pièce : placé au cœur d'un poème en manière de cadavre exquis, écrit par La Petite sur une feuille blanche, qui s'ouvre et se termine par la formule "Les pierres vous donnent des oiseaux », la pierre donne au récit la dimension spéculaire d'un éternel recommencement, où se rejoue sans cesse la même situation dramatique qui fait retour invariablement sur le plateau. Principe organisateur de l'intrigue, le caillou est donc bien le fil rouge d'une dramaturgie diffractée, bien que spéculaire. 

est encore plus complexe, puisqu'elle prévoit plusieurs actions simultanées ( On peut imaginer que les séquences sept et huit se jouent en même temps", p. 55). Elle envisage différentes combinaisons possibles des «séquences» au sein d'un dispositif scénographique qui exploite les accessoires du conte-source en leur conférant une portée symbolique et métaphysique renouvelée : «Il y a toujours quelque part un chemin. Parfois ce chemin traverse la mer, les gratte-ciels, des arbres, et bien d'autres choses encore. [...] Sur le plateau, il y a simplement une porte et sept cailloux blancs. Avec ça la lumière, qui met en valeur les contrastes, accentue la beauté, guide la petite fille sur son chemin. » (p. 34) L'intrigue a peu de rapport avec le conte de départ : elle articule un récit-cadre où une petite fille apporte un cadeau à une autre, malade et confinée à l'hôpital, à l'histoire d'une Petite Poucet qui, pour échapper à l'emprise parentale, entreprend à l'aide de sept cailloux magiques un voyage initiatique avec ses sœurs en cherchant de toutes ses forces à échapper à l'emprise trop aimante de ses parents. Extrapolant à partir des motifs merveilleux du conte, la pièce en inverse ainsi le schéma d'abandon au moyen de la substitution entre parents absents et parents trop présents et de la réhabilitation de l'ogre aux pouvoirs de séduction, par le truchement du personnage du docteur Logre.

plateau de " cailloux qui sont des cailloux » (p. 34), la scénographie fonctionne donc comme une sorte de citation visuelle du contesource, qui n'est explicitement sollicité dans le dialogue qu'à une seule reprise, sous forme de mise en abyme, pour donner une explication crédible à la divagation poétique de la jeune fille : «Le Petit Poucet a toujours été son conte préféré» (p. 66). Les cailloux sont présents sur scène avant même le début de l'action, comme l'indique la didascalie inaugurale: "Porte fermée, les sept cailloux sont dans un coin» (Un, p. 35). Puis ils réapparaissent de façon relativement systématique au début de chaque séquence, donnant lieu à différents jeux de scène. Plus fondamentalement, ils matérialisent la signification implicite de l'action : "Petite Poucet dessine un chemin avec ses cailloux blancs, en direction de la porte » (Deux, p. 39). Elle est encore soulignée par le refrain polyphonique répété et amplifié tout au long de la pièce comme une ritournelle obsessionnelle :

À quoi ils servent tes cailloux.

À faire le chemin.

T'iras pas loin avec sept cailloux.

Si. Avec sept cailloux j'irai partout.

(Trois, p. 42 et suiv.)

C'est bien d'une échappée volontaire qu'il s'agit dans ce conte dramatique plutôt que du traditionnel schéma d'abandon. Les «cailloux de la rivière de l'été », blancs, volés dans la jardinière de la mère, marquent le chemin à suivre pour s'émanciper de déterminismes familiaux et sociaux, suggérant la finalité implicite de l'action rêveuse de la petite fille, qui n'a de cesse de quitter un univers marqué par le trop-plein (« Elle a tout ce qu'il faut ici. Tous les jouets, tout l'amour, tout ", Sept, p. 52), à seule fin de se confronter à un monde où « il y a trop de bruit, trop d'air, trop de tout. On ira voir tout »; « On habitera la vraie vie » (p. 39-40). Ils revêtent également une dimension symbolique, comme lorsque la Petite Poucet procède à la distribution à ses sept sœurs, qui portent toutes des noms de fleurs, et leur dit : "Après sept, plus rien possible, huitième caillou existe pas. " (Cinq, p. 48) Ils prennent enfin une dimension mythologique, voire cosmogonique, lorsque la Petite Poucet est mise en demeure par ses sœurs, qui décidément ne « comprennent pas les cailloux ", de s'expliquer sur le jeu consistant à substituer des cailloux les uns aux autres en avançant :

ILCEA, 20 | 2014 
On suit les cailloux. Les cailloux, c'est nos pas dans la terre, dans le sable, sur les rochers, sur le pavé, les cailloux c'est pour aller loin. Des fois on les jette devant nous et on saute, comme à la marelle. Des fois on les pose les uns sur les autres pour traverser la rivière. Pour voir de l'autre côté du mur. Des fois on les colle les uns aux autres et ça fait une allée pour pas se salir les pieds. Des fois on fait du feu avec. Des fois on les pose sur nos genoux et on mange la soupe dedans. Des fois on les met sur la tête pour se protéger de la grêle. Des fois on écrase un scorpion une araignée un sanglier. Des fois on les lance très haut et les pommes et les poires tombent dans nos mains. Des fois on se frotte les jambes avec et on est toutes propres. Des fois on les fait chauffer au soleil et on les tient sur son ventre quand on a mal. Des fois on les met dans ses poches et on s'enfonce dans l'eau profonde. Des fois on les pose sur un cœur. Des fois on les tient dans sa main on les embrasse on les caresse. Des fois on les met sur ses yeux pour les voir. Des fois on pose sa tête sur leur oreiller. Des fois on les mouille et on se regarde dedans. On fait son chemin. On va loin. On va sept fois plus loin. (Neuf, p. 59-60)

Le caractère hypnotique de l'allitération donne une dimension rituelle au geste consistant à déplacer le caillou, évoqué pour convoquer le monde sur le plateau et embrayer la nouvelle odyssée poétique de la Petite Poucet. Il fait écho aux derniers mots de la pièce, où la sempiternelle ritournelle s'achève sur une assertion rompant le cycle de la narration : «AVEC SEPT J'IRAI PARTOUT. » (Douze, p. 67)

\section{Auteurs en quête de personnage}

$$
\begin{aligned}
& \text { J'ai laissé la fin s'imposer d'elle-même, } \\
& \text { dans toutes les couleurs de l'arc-en-ciel. } \\
& \text { Une éclaircie dans la forêt, } \\
& \text { une trêve avec soi-même et avec le monde... } \\
& \text { (p. 81) }
\end{aligned}
$$$$
\begin{aligned}
& \text { L'Ogrelet, affichant une désinvolture apparent } \\
& \text { résolution du conflit dramatique qui s'est noué }
\end{aligned}
$$

Tel est également le sens du « livre blanc» tendu par la Dévoreuse de livres à Alia dans Mange-moi de Nathalie Papin : «Tiens, c'est immangeable ! Il n'y a rien dedans, il n'y a rien d'écrit. Ça peut être utile à ton ogre. Il n'a qu'à écrire là-dedans ce qu'il veut. [...] Écrire une histoire à deux, c'est pas facile. Faut essayer ! (p. 70) Métaphore de la lecture, le livre blanc qu'on écrit à deux est également une parabole de l'art du récit en tant que tel et de sa vocation heuristique : «Ça c'est pour la suite. Pour faire ton histoire, pour devenir ce que tu veux devenir. » (p. 74-75)

C'est pourtant chez Philippe Dorin, dans En attendant le Petit Poucet, que le double mouvement de prise de distance et d'exemplification du conte-source est le plus sensible. 
À l'instar des pages blanches du conte philosophique de Voltaire avec Micromégas, le dénouement de la pièce prend une portée allégorique et métathéâtrale essentielle, tout comme l'hommage à Samuel Beckett et l'évocation de la crise du drame suggérés par le titre lui-même : alors qu'ils se croyaient perdus, les enfants, mimant pour tromper l'ennui et la peur l'histoire de Poucet et l'arrivée dans la maison de l'Ogre, se trouvent confrontés à une curieuse découverte : "Dans cette maison, il y a justement quelqu'un qui cherche désespérément une histoire. Voilà des mois qu'il tourne en rond, lui aussi. Il n'attend plus que vous. Votre chambre est prête. Il vous a préparé un lit, dans la pièce à côté. » (Scène 5, p. 72) C'est en réalité à "une page blanche» que les enfants sont finalement confrontés, ce qui suscite la réticence du Grand (« Mais je ne peux pas entrer comme ça dans une page blanche!»), mais également la réserve de la Petite, désireuse de ne pas se séparer de son fameux caillou anthropomorphe («Les cailloux, ça tient pas dans les pages! »), quitte à donner de l'humeur à son frère : « Arrête de trembler comme ça, tu vas froisser la page » (p. 73-74)...

Dès lors, le conte merveilleux n'est plus un patrimoine culturel fécond, autorisant une infinité de variations rétrospectives en cent actes divers, mais l'espace de projections et d'expérimentations scéniques prospectives. Il constitue surtout l'horizon d'attente impossible d'un théâtre contemporain qui puise en lui les ressources de son propre décentrement, voire de l'exténuation de la fable et du mythe. Le conte merveilleux, en révélant une incomplétude qui est aussi celle de l'existence elle-même, apparaît dès lors plus que jamais fécond, jusque dans sa propre dénégation...

\section{BIBLIOGRAPHIE}

\section{Euvres étudiées}

BARAtoux Caroline (2008), Le Petit Poucet, illustrations de Vincent Fortemps, Arles : Actes SudPapiers.

CECCONI-BOTELla Monic (composition) et Fleutiaux Pierrette (livret), La Femme de l'ogre, opéraconte, France Culture - service de la création musicale - atelier de la création radiophonique, première diffusion à l'automne 1989.

DORIN Philippe (2001), En attendant le Petit Poucet, Paris : L'École des loisirs.

GALEA Claudine (2009), Petite Poucet, Montpellier : Espaces 34.

GRANGEAT Simon, Un Caillou dans la botte. Histoire du Petit Poucet racontée par l'Ogre, texte non publié à ce jour mais dont le manuscrit est déposé à la SACD.

GRANOUILLET Gilles (2012), Poucet pour les grands, Manage, Belgique : éditions Lansman.

GRUMBERG Jean-Claude (2006), Mange ta main. Conte pour enfants précoces et adultes attardés, Arles : Actes Sud Papiers.

GUTMANN Laurent, Petit Poucet, ou Du Bienfait des balades en forêt pour l'éducation des enfants, créé au Théâtre des Cinq Diamants à Paris en février 2012. 
LEBEAU Suzanne (1997), L'Ogrelet, Paris : Théâtrales.

MURGIA Fabrice (2010), Le Chagrin des ogres, Carnières / Morlanwelz, Belgique : Lansman éditeur.

PAPIN Nathalie (2002), Mange-moi, Paris : L'École des loisirs.

PLIYA José (2011), Mon Petit Poucet, Paris : L'avant-scène théâtre.

\section{Critiques et analyses}

AUROUET Carole (dir.) (2005), Contes et légendes à l'écran, Paris : Corlet éditions.

BAHIER-PORTE Christelle (dir.) (2007), « Le conte à la scène : enquête sur une rencontre », Féeries, 4. BARKER Howard (2009), « Ces tristes lieux, pourquoi faut-il que tu y entres?», Arles : Actes Sud. BARTHES Roland (1957), « Le mythe aujourd'hui », Mythologies, Paris : Seuil.

BAUMGARDT Ursula et BORNAND Sandra (dir.) (2009), « Autour de la performance », Cahiers de Littérature Orale, 65.

BELMONT Nicole (1999), Poétique du conte (essai sur le conte traditionnel oral), Paris : Gallimard.

BENJAMIN Walter (1991), « Le Narrateur », Écrits français, Paris : Gallimard.

BENJAMIN Walter (2000), « Le Conteur », Euvres III, Paris : Gallimard.

BERNANOCE Marie (2006), À la découverte de cent et une pièces. Répertoire critique du théâtre contemporain pour la jeunesse, Montreuil-sous-Bois / Grenoble : Éditions théâtrales / SCEREN.

BERNANOCE Marie (2007), «L'adaptation dans le théâtre jeunesse : contre l'esthétique du débarras ", Adapter des œuvres littéraires pour les enfants, Grenoble : SCEREN.

BERNANOCE Marie (2008), «Les réécritures de conte dans le théâtre contemporain pour les jeunes : un nouveau regard sur les relations familiales? », C. d'Humières (dir.), D'un conte à l'autre, d'une génération à l'autre, Clermont-Ferrand : Presses de l'Université Blaise Pascal.

BERNANOCE Marie (2012), Vers un théâtre contagieux, Montreuil : Théâtrales.

BeTtelheim Bruno (1999), Psychanalyse des contes de fées (Th. Carlier, trad.), Paris : Robert Laffont 1976, réédition Pocket.

COLLECTIF (2003), Théâtre Aujourd'hui, 9, « Théâtre et enfances : l'émergence d'un répertoire », CNDP.

DEFRANCE Anne \& PERRIN Jean-François (dir.) (2007), Le Conte en ses paroles. La figuration de l'oralité dans le conte merveilleux du Classicisme aux Lumières, Paris : Desjonquères.

DOUHOURE Vaber \& BOST Bernadette (dir.) (2004), Le Renouveau du conte : l'exemple du conte spectaculaire, Grenoble : ELLUG.

GAYOT Joëlle \& POMMERAT Joël (2009), Joël Pommerat, troubles, Arles, Actes Sud Papiers.

LA SALLE Bruno de (1995), Le Conteur amoureux, Paris : Casterman.

LA SALLE Bruno de (2004), Plaidoyer pour les Arts de la parole, Vendôme : Centre de Littérature Orale (Clio), p. 42.

LA SAlle Bruno de \& Gougaud Henri (2002), Le Murmure des contes, Paris : Desclée de Brouwer.

LA SAlLE Bruno de, Jolivet Michel, touATI Henri \& CRANSAC Francis (dir.) (2005), Pourquoi faut-il raconter des histoires? Paris : Éditions Autrement / MondOral, vol. I. 
LA SAlle Bruno de, Jolivet Michel, touati Henri \& CRANSAC Francis (dir.) (2006), Pourquoi faut-il raconter des histoires? Paroles de conteurs, Paris : Éditions Autrement / MondOral, vol. II.

LAPLACE-CLAVERIE Hélène (2007), Modernes Féeries : Le théâtre français du XXe siècle entre réenchantement et désenchantement, Paris : Champion.

MARTIN Roxane (2007), La Féerie romantique sur les scènes parisiennes (1791-1864), Paris : Champion.

MURGIA Fabrice (2009), « Exprimer un état d'esprit », entretien réalisé par Nancy Delhalle, Alternatives Théâtrales, 100, « Poétique et politique ».

PIFFAUlt Olivier (dir.) (2001), Il était une fois... les contes de fées, Paris : Seuil / BnF.

POIRSON Martial (dir.) (2012), "Le conte à l'épreuve de la scène contemporaine (XX-XXI ${ }^{\mathrm{e}}$ siècles) ", Revue d'Histoire du théâtre, 1 et 2.

POIRSON Martial (dir.) (2009), Perrault en scène : transpositions dramatiques de contes merveilleux (1697-1800), Montpellier : Espaces 34.

POIRSON Martial (2011), « Le conte merveilleux, ouvroir de littérature dramatique potentielle : le cas des transpositions théâtrales du Petit Poucet de Perrault », M. Poirson et J.-Fr. Perrin (dir.), Les Scènes de l'enchantement : arts du spectacle, théâtralité et conte merveilleux (XVII-XIXe siècles) (133-175), Paris : Desjonquères.

POMMERAT Joël (2007), Théâtres en présence, Arles : Actes Sud Papiers.

PROPP Vladimir (1970), Morphologie du conte, Paris : Seuil.

SARRAZAC Jean-Pierre (1999), L’Avenir du drame, Paris : Circé.

SARRAZAC Jean-Pierre (2002), La Parabole ou l'enfance du théâtre, Paris : Circé.

SORIANO Marc (1978), Les Contes de Perrault. Culture savante et traditions populaires, Paris : Gallimard. TOUATI Henri (2000), L'Art du récit en France : état des lieux, problématique, Ministère de la Culture, Direction de la Musique, de la Danse, du Théâtre et des Spectacles.

TRAORE Aminata (2002), Le Viol de l'imaginaire, Paris : Fayard.

\section{Filmographie}

Du conte au théâtre, avec la compagnie Louis Brouillard, DVD, SCÉRÉN, 127', février 2008.

BoISRond Michel (1972), Le Petit Poucet, 1 h 30, avec Marie Laforêt, Jean-Pierre Marielle, Jean-Luc Bideau.

DAHAN Olivier (2007), Le Petit Poucet, Studio Canal, 127', dans des décors de Régis Loisel, avec Romane Bohringer, Élodie Bouchez, Samy Naceri, Catherine Deneuve et Saïd Taghmaoui. DE VAN Marina (2010), Le Petit Poucet, 142', avec Denis Lavant, Ilian Calabert, Rachel Arditi, Adrien De Van.

\section{Sitographie}

Site d'information théâtrale, en ligne sur <www.theatre-contemporain.net/spectacles/>.

Conservatoire contemporain de littérature orale, en ligne sur <www.clio.org/>.

Revue Féeries, en ligne sur <http://feeries.revues.org/>.

La Grande Oreille, La revue des arts de la parole, en ligne sur <www.lagrandeoreille.com/>. 
Cahiers de littérature orale, en ligne sur <http://clo.revues.org/>.

Note d'intention sur Le Petit Poucet, ou Du Bienfait des balades en forêt pour l'éducation des enfants, en ligne sur <www.theatre-5-diamants.fr/programmation-fiche-theatre/-le-petit-poucet-,10>.

Note d'intention sur Mon Petit Poucet, en ligne sur <www.varia.be/upload/relationspubliques-2011-2012/Monpetitpoucetdossierpedagogique.pdf>.

Dossier d'accompagnement de Poucet pour les grands, en ligne sur <www.theatreleverso.com/IMG/ pdf/Poucet_dossier_nov_2012.pdf>.

Interview de Gilles Grenouillet, en ligne sur <www.dailymotion.com/video/xt6e8v_poucet-pourles-grands_creation>.

Dossier d'accompagnement d'Un Caillou dans la botte, en ligne sur <www.simongrangeat.fr/ archives/1125>.

Note d'intention sur Le Chagrin des ogres, en ligne sur <www.theatre-contemporain.net/ spectacles/Le-Chagrin-des-ogres>.

Note d'intention sur la mise en scène de Le Petit Poucet par Pascale Nandillon, en ligne sur < www.atelierhorschamp.org/Spectacles/Archivage/poucet_resu.html>.

\section{NOTES}

1. Dossier du spectacle, p. 6. Dans l'ensemble de cet article, les références aux pièces publiées et aux articles en ligne sont mentionnées à l'occasion de leur première occurrence, les indications de pagination qui suivent renvoyant à cette édition.

2. Ainsi notamment d'O. Dahan dans Le Petit Poucet, Studio Canal, 2007, 87', après M. Boisrond dans Le Petit Poucet, 1972, 1h30. La dernière adaptation en date par Marina De Van avec Le Petit Poucet, en 2011, relève davantage du film fantastique que de la transposition.

3. Créé au Théâtre des Cinq Diamants à Paris en février 2012. Note d'intention en ligne.

4. Je remercie Henri Touati, directeur des Arts du Récit en Isère, d'avoir attiré mon attention sur cette source issue de la culture orale et de m'avoir sensibilisé à ces traditions d'artistes-conteurs. 5. J. Pliya, Mon Petit Poucet, 2011, préface: «Le Paradis tragique des enfants », p. 7. Créé au Théâtre Varia / Centre dramatique de la communauté française Wallonie-Bruxelles en 2011.

6. Le spectacle n'hésite pas à franchir allègrement le quatrième mur, comme dans L'Ogre déchu ou Le Savoir des plus petits, proposé par la compagnie italienne du Teatro delle briciole au Théâtre de Genève en 2001, où un comédien, qui incarne tour à tour le Père, l'Ogresse et l'Ogre, organise l'« enlèvement » de trois enfants pris au hasard au sein du public pour interpréter Poucet et ses frères.

7. Créé par Marie Mainardis et Catherine Salvini au Théâtre aux Mains Nues (Paris) en mai 2004, puis au Thalia Theater de Halle (Allemagne) en 2008.

8. Dossier du spectacle en ligne.

9. Dossier du spectacle en ligne.

10. Créé par la compagnie Travelling théâtre au Théâtre Nouvelle Génération de Lyon et à l'Opéra-Théâtre de Saint-Étienne en novembre 2012.

11. Dossier du spectacle en ligne.

12. La Femme de l'ogre, opéra-conte, musique de M. Cecconi-Botella, livret de P. Fleutiaux, France Culture - service de la création musicale - atelier de la création radiophonique, première diffusion à l'automne 1989.

13. Créé par Clément Arnaud et la compagnie Traversant 3 à la MJC Jean Macé de Lyon en octobre 2011. Note d'intention, p. 2. 
14. S. Grangeat, Un Caillou dans la botte, canevas de la pièce joint au dossier d'accompagnement du spectacle, déjà mentionné, p. 2.

15. Créé par Gervais Gaudreault et le Carroussel à l'Espace Malraux de Chambéry en octobre 1997, puis par Christian Duchange au Théâtre de la Commune d'Aubervilliers en mars 2008, avec Géraldine Pichon et Pascal Delannoy.

16. Créé au Théâtre National de Bruxelles et au Festival de Liège en 2009, repris au Théâtre de l'Odéon en 2010. Note d'intention en ligne.

17. L. Gutmann (2012), note d'intention.

18. P. Nandillon, note d'intention sur la mise en scène de Le Petit Poucet, en ligne sur < www.atelierhorschamp.org/Spectacles/Archivage/poucet_resu.html>.

\section{RÉSUMÉS}

Depuis une vingtaine d'années, le conte-source du «Petit Poucet» de Charles Perrault fait son retour, sous des formes souvent détournées, dans le théâtre francophone contemporain, preuve de son acuité politique, de sa fécondité dramaturgique et de sa plasticité scénographique. C'est au prix d'une transformation notable et significative: de progéniture, Poucet devient géniteur, faisant basculer le conte du récit initiatique de formation vers la fable de transmission. En s'affranchissant des règles de l'adaptation et en prenant leurs distances par rapport au patrimoine culturel, les transpositions scéniques du «Petit Poucet " tendent à démultiplier les points de vue et à évacuer la portée axiologique du conte au profit d'une épicisation de la fable et d'une amplification métaphorique susceptibles d'exhiber la théâtralité inhérente au contesource, tout en rendant visible la structure autoréférentielle du dispositif spectaculaire. Elles donnent une dimension générique au personnage éponyme et développent le motif central de la dévoration afin d'exacerber l'ambivalence constitutive du conte. Cet article envisage la façon dont les transpositions théâtrales, en se démarquant du conte-source, proposent une forme d'actualisation, voire de déterritorialisation dysfonctionnelle susceptible d'en révéler la teneur idéologique, mais également d'en réamorcer la charge symbolique, tout en révélant ses potentialités dramaturgiques et scéniques.

For the past twenty years, one of the best-known traditional French tales known as "Le Petit Poucet" (Little Thumbling) by Charles Perrault has been more and more present in the art world. The reason for this "cultural comeback", especially in modern Francophone theater, might be seen as indicative of the tale's political sharpness, dramaturgical possibilities and malleable aspect of its scenography. However, the retelling of this classical tale shows a major change from the original story as Little Thumbling is not only presented as a child, but also as a father himself. This significant change therefore affects the very nature of the tale which goes beyond the story of a boy in the process of learning and growing, but of someone passing on his knowledge.

The plot no longer simply highlights the values of a society but, as stage adaptations have shown, it also serves as a means to offer various points of view. Hiding some of his individual characteristics, the main character (Petit Poucet) becomes more and more generic to allow the reader to self-identify with the protagonist. The central theme of cannibalism itself-an ogre eating children-, is amplified and shown in a concrete (rather than just metaphorical) way. This article aims to show that contemporary stage adaptations of "Le Petit Poucet" not only update the classical tale but also highlight the ambivalence of the story and its ideological subtext. 
INDEX

Keywords : tale, Perrault, « Petit Poucet »/Little Thumbling, contemporary French theatre, adaptation, rewriting, stage adaptation, actualization, de-territorialisation, mythologisation, dramaturgy, scenography, staging, meta-theatrical Mots-clés : conte, Perrault, Petit Poucet, théâtre contemporain francophone, adaptation, réécriture, transposition théâtrale, actualisation, déterritorialisation, mythologisation, dispositif esthétique et idéologique, dramaturgie, scénographie, mise en scène, métathéâtral

\section{AUTEUR}

MARTIAL POIRSON

Université de Paris 8 - Saint Denis 